\title{
Methoxylation enhances stilbene bioactivity in Caenorhabditis elegans
}

\author{
Mark A Wilson ${ }^{1}$, Agnes M Rimando ${ }^{2}$ and Catherine A Wolkow*1
}

Address: ${ }^{1}$ Laboratory of Neurosciences, National Institute on Aging, Intramural Research Program, NIH, Baltimore, MD 21224, USA and ${ }^{2}$ Natural Products Utilization Research Unit, ARS, US Department of Agriculture, PO Box 8048, University, MS 38677, USA

Email: Mark A Wilson - wilsonma@grc.nia.nih.gov; Agnes M Rimando - agnes.rimando@ars.usda.gov;

Catherine A Wolkow* - wolkowca@grc.nia.nih.gov

* Corresponding author

Published: 13 August 2008

BMC Pharmacology 2008, 8:15 doi:10.1186/147|-2210-8-15
Received: II June 2008

Accepted: 13 August 2008

This article is available from: http://www.biomedcentral.com/I47/-22/0/8/15

(C) 2008 Wilson et al; licensee BioMed Central Ltd.

This is an Open Access article distributed under the terms of the Creative Commons Attribution License (http://creativecommons.org/licenses/by/2.0), which permits unrestricted use, distribution, and reproduction in any medium, provided the original work is properly cited.

\begin{abstract}
Background: Stilbenes are 1,2-diphenylethylene congeners produced by plants in response to stress. Many stilbenes also exhibit xenobiotic activities in animal cells, such as inhibition of cancer cell growth, neuroprotection, and immune modulation. In vivo, hydroxylated stilbenes are metabolized by glucuronidation to facilitate excretion. Methoxylated stilbenes are metabolized more slowly, which may have a positive effect on in vivo bioactivity. Here, we have directly compared in vivo bioactivities of methoxylated and hydroxylated stilbenes in a whole organism using the roundworm Caenorhabditis elegans, an advantageous experimental system for such studies due to its rapid lifecycle, genetic amenability and relatively low-cost.
\end{abstract}

Results: Toxicity towards $C$. elegans adults was observed for trimethoxylated and dimethoxylated stilbenes, as well as the monomethoxylated stilbene desoxyrhapontigenin. Toxicity was not observed for the monomethoxylated stilbene, pinostilbene, nor for hydroxylated stilbenes. The methoxylated stilbenes that exhibited toxicity also showed stronger inhibitory effects than the hydroxylated stilbenes on germline tumor growth in gld-I(q485) adults. However, steady-state levels of three inhibitory methoxylated stilbenes did not directly correlate to their relative bioactivities.

Conclusion: These findings demonstrate that, for the group of stilbenes investigated, methoxylation generally increased bioactivity in vivo in a whole organism, with the exception of pinostilbene. Differences in bioactivity in C. elegans adults did not appear to correlate with differential uptake. Rather, we speculate that methoxylated stilbenes may have increased interactions with biological targets in vivo or may interact with specific targets unaffected by hydroxylated stilbenes. The potent activities of methoxylated stilbenes provide a basis for further investigations to identify in vivo targets for these compounds.

\section{Background}

Phytochemicals induce an array of biological responses in animal cells, including enzyme inhibition, protection against oxidation and regulation of cellular signaling pathways. Among phytochemicals with xenobiotic activities, the stilbenes are of great interest for their potential medical impact. Stilbene compounds, particularly pterostilbene and resveratrol, have been associated with anti- 
diabetic, anticarcinogenic and antilipogenic activities in vitro and in vivo [1-4]. Specific enzymatic targets are known for some of these xenobiotic activities. Pterostilbene and related compounds inhibit the cytochrome P450 enzymes, CYP1A1 and CYP1B1, which induce carcinogenicity of environmental teratogens [5]. Pterostilbene also inhibits cyclooxygenases COX-1 and COX-2, interfering with endocrine functions and supporting analgesic activity [6]. Significant increase in activity of hexokinase, while significant decrease in activity of glucose-6-phosphatase and fructose-1,6-bisphosphatase, were observed after oral administration of pterostilbene in diabetic rats [7]. The related compound, resveratrol, stimulates sirtuin enzymes to promote longevity in response to dietary restriction $[8,9]$. Resveratrol has also been demonstrated to protect against obesity- and diet-related disease in rodents [10]. Additional interactions with new targets may be found to underlie other activities of these compounds.

While dietary stilbenes and flavonoids are of particular interest for their potential health benefits, in vivo activity is limited by low bioavailability due to rapid metabolism and excretion. Studies have found that methoxylation can protect flavonoids from derivatization, thereby improving biostability $[11,12]$. However, there is also evidence that methoxylation can alter bioactivity. For example, methoxylated flavones induced different cell-cycle arrest points in cultured human cells than hydroxylated flavones [13]. Thus, methoxylation may alter interactions with target proteins, as well as increase bioavailability. These reports on altered bioactivity in methoxylated flavonoids triggered our interest to investigate the effects of methoxylation on stilbene bioactivity, particularly in a whole organism, to assess effects on postmitotic cells in adult tissues as well as on actively dividing cells.

Use of invertebrates provides one approach to address these questions rapidly and economically in a whole organism. In particular, the roundworm, Caenorhabditis elegans, offers many advantages for such analyses. C. elegans has a short generation time of 1 week and are easily maintained in large numbers with ordinary laboratory equipment. The adult body is approximately $1 \mathrm{~mm}$ in length, and contains most of the tissues present in higher vertebrates, such as a complete nervous system, striated muscles and an intestine with digestive, detoxification and innate immune functions. It is of note that the adult somatic tissues are post-mitotic and therefore lack cellreplacement capacity. C. elegans are hermaphrodites and able to self-fertilize, which has greatly facilitated the genetic analysis of developmental and cell biological processes in this organism. Previous uses of C. elegans for pharmacological studies have taken advantage of mutant strains for mechanistic studies, in addition to basic studies of compound-induced gene expression or phenotypic changes [14-16].

In our study, we used C. elegans to compare the bioactivities of methoxylated and hydroxylated stilbenes, based on the parent compound resveratrol. Seven stilbenes differing in hydroxylation and methoxylation patterns were examined for effects on survival of fem-1(hc17) sterile adults and growth of germline tumors in gld-1(q485) mutants. Methoxylated stilbenes exhibited greater bioactivity in both assays, as compared with hydroxylated stilbenes. Steady-state levels for three of these compounds (one mono-, one $\mathrm{di}_{-}$-, and one tri-methoxylated) were measured in treated worms and were not found to correlate with relative bioactivity. This suggests that the greater bioactivity of methoxylated stilbenes does not reflect differential uptake of the compounds. These findings demonstrate that methoxylation substitution enhances stilbene bioactivity in this whole-organism model. Methyl group-specific target site interactions may be one factor accounting for the differential bioactivities of these compounds. Alternatively, methoxylation may protect stilbenes from metabolic modification and excretion, leading to higher potency in vivo in C. elegans than hydroxylated stilbenes.

\section{Results and discussion}

Seven methoxylated or hydroxylated stilbenes were compared for effects on C. elegans survival and tumor growth. This collection included two hydroxylated structures (resveratrol and piceatannol), two monomethoxylated structures (pinostilbene and desoxyrhapontigenin), two dimethoxylated structures (3-hydroxy-5,4'-dimethoxystilbene and pterostilbene) and one trimethoxylated structure (resveratrol-trimethylether) (Figure 1). Bioactivity in



\section{Figure I}

Structures of methoxylated and hydroxylated stilbenes tested in this study. 
C. elegans has been reported only for resveratrol, which extended adult lifespan [9]. It was of interest, therefore, to compare adult survival in animals treated with resveratrol and the related compounds.

For survival assays, young adult (day 0) animals were transferred onto fresh medium supplemented with the test compound at a range of concentrations. The compounds were tested at indicated concentrations in the growth medium (below). C. elegans nematodes are surrounded by a thick collagenous cuticle and are therefore relatively impermeable to environmental compounds. Consistent with this, our measurements demonstrated that internal concentrations of these compounds were significantly lower than their concentrations in the medium. Survival was scored every 2-3 days as the ability to move in response to a gentle mechanical stimulus. During the first week of adulthood, C. elegans hermaphrodites are reproductively competent and normally produce 200300 progeny. Since progeny production would complicate survival analysis, these experiments were performed using fem-1 (hc17) animals that are sterile due to a spermatogenesis defect $[17,18]$. Our prior analyses have confirmed that fem-1(hc17) and wildtype animals have similar lifespan characteristics [15].

Under our experimental conditions, both hydroxylated and one monomethoxylated stilbene, pinostilbene, had negligible or modestly beneficial effects on adult survival (Figure 2). Consistent with previous reports, we observed that resveratrol had a modestly beneficial effect on adult lifespan $(0-15 \%$ at $100 \mu \mathrm{M}$ dose) (Table 1$)$. However, the effect was variable and did not attain statistical significance in our experiments. In addition, piceatannol and pinostilbene had beneficial effects on lifetime survival. The effects of these compounds were also modest (5$15 \%)$ and were not statistically significant.

The methoxylated stilbenes, desoxyrhapontigenin, 3hydroxy-5,4'-dimethoxystilbene, pterostilbene and resveratrol-trimethylether, each exhibited significant detrimental effects on adult survival (Figure 3). At a $100 \mu \mathrm{M}$ dose initiated on the first day of adulthood, survival was reduced $11-26 \%$ by desoxyrhapontigenin, $48-57 \%$ by $3-$ hydroxy-5,4'-dimethoxystilbene, $21-28 \%$ by pterostilbene and $18-51 \%$ by resveratrol-trimethylether (Table 1 ). The toxicity of resveratrol-trimethyether increased steeply within a relatively limited dose range, between 5-20 $\mu \mathrm{M}$. In contrast, the toxicity of desoxyrhapontigenin, 3hydroxy-5,4'-dimethoxystilbene and pterostilbene gradually increased between the $20-100 \mu \mathrm{M}$ doses. The observed difference in activity between the monomethoxylated stilbenes, desoxyrhapontigenin being toxic while pinostilbene not, may be due to differences in steric hinderance introduced by the bulky methoxy groups at different positions in the stilbene ring. Without knowing the target site(s) in C. elegans for these stilbenes, it can be speculated that the position of the methoxy group at ring A in pinostilbene causes unfavourable fit for target site inhibition. Differences in activity of these monomethoxylated stilbenes have been demonstrated in other studies; e.g., pinostilbene moderately inhibited the catalytic activity of cytochrome P450 2E1, while desoxyrhapontigenin was not inhibitory [19]. We noted that animals treated with either methoxylated or hydroxylated stilbenes did not display any other noticeable phenotypes, such as altered feeding or movement rates.

The somatic tissues of C. elegans adults are comprised solely of post-mitotic cells and lack the ability to renew by stem cell replacement. To more closely examine the effects of methoxylated stilbenes on mitotically-active cells in this organism, we examined their effects on growth of germline tumors produced in gld-1(q485) animals. The $C$. elegans hermaphrodite gonad is a U-shaped structure that is bisymmetric and contains the germ cell pool (Figure 4A, gld-1(+)). Within each gonad arm, germ cells in the distal region undergo mitotic proliferation in response to Notchlike signals produced by cells at the distal tips of the gonad arms. As germ cells migrate along the gonad arm, they exit from mitosis, undergo meiosis and subsequently differentiate into mature oocytes. In gld-1(q485) mutants, the germ cells fail to exit from mitosis and continue to proliferate throughout the gonad forming a germline tumor that is lethal to the animal (Figure 4A, gld-1(q485)) $[20,21]$.

Several stilbenes have demonstrated antiproliferative effects on mammalian cancer cells in vitro [22-25]. To assess the effects of methoxylation on antitumor activity in vivo, tumor area was measured in gld-1(9485) adults treated for four days with either resveratrol trimethylether, desoxyrhapontigenin or pterostilbene, which are methoxylated and toxic to adults, or resveratrol, which is hydroxylated and nontoxic. As controls, germline tumor area was also measured in animals treated with carrier only (ethanol) or with 5-fluoro-2'-deoxyuradine (FUDR), a thymidylate synthetase inhibitor that blocks cell proliferation $[26,27]$. FUDR was chosen as a positive control for its known antiproliferative effects in C. elegans, which have been demonstrated by inhibition of embryonic and larval development [28]. After four days of treatment, animals were collected and germline tumors were visualized by DAPI staining to visualize germcell nuclei. Because the level of DAPI staining was not quantitative between animals, we DAPI signal intensity was not used as a measure of tumor size. Rather, tumor size was directly measured as the area of the DAPI-stained gonad.

For the initial tests of tumor growth suppression, each compound was tested at a $100 \mu \mathrm{M}$ dose in the growth medium (Figure 4B). At this concentration, FUDR exhib- 
Table I: Adult survival statistics for all trials.

\begin{tabular}{|c|c|c|c|c|c|c|}
\hline \multirow[t]{2}{*}{ Trial } & \multirow[t]{2}{*}{ Concentration $(\mu \mathrm{M})$} & \multicolumn{2}{|c|}{ Mean lifespan (days) } & \multirow[t]{2}{*}{ Relative survival (\%) } & \multirow[t]{2}{*}{ p (Logrank) } & \multirow[t]{2}{*}{ n (failed, censor) } \\
\hline & & Treated & Control & & & \\
\hline \multicolumn{7}{|c|}{ Resveratrol-trimethylether } \\
\hline 1 & 100 & 9.7 & 12.16 & $80 \%$ & 0.004 & 61,0 \\
\hline 4 & 20 & 7.72 & 11.26 & $69 \%$ & $<0.001$ & 61,0 \\
\hline 4 & 40 & 7.72 & 11.26 & $69 \%$ & $<0.001$ & 74,0 \\
\hline 4 & 100 & 7.18 & 11.26 & $64 \%$ & $<0.001$ & 70,2 \\
\hline 5 & 5 & 18.83 & 18.49 & $102 \%$ & 0.91 & 46,3 \\
\hline 5 & 20 & 16.41 & 18.49 & $89 \%$ & 0.055 & 42,3 \\
\hline 5 & 100 & 15.18 & 18.49 & $82 \%$ & 0.005 & 37,6 \\
\hline 6 & 20 & 9.02 & 15.32 & $59 \%$ & $<0.0001$ & 49,5 \\
\hline 6 & 40 & 7.98 & 15.32 & $52 \%$ & $<0.0001$ & 51,0 \\
\hline 6 & 100 & 7.54 & 15.32 & $49 \%$ & $<0.0001$ & 52,0 \\
\hline \multicolumn{7}{|c|}{ 3-OH-5,4'- dimethoxystilbene } \\
\hline 1 & 100 & 6.34 & 12.16 & $52 \%$ & $<0.0001$ & 53,0 \\
\hline 2 & 20 & 11.05 & 11.05 & $100 \%$ & 0.986 & 47,2 \\
\hline 2 & 40 & 10.52 & 11.05 & $95 \%$ & 0.234 & 40,1 \\
\hline 6 & 20 & 10.38 & 15.32 & $68 \%$ & $<0.0001$ & 56,0 \\
\hline 6 & 40 & 7.68 & 15.32 & $50 \%$ & $<0.0001$ & 54,1 \\
\hline 6 & 100 & 6.56 & 15.32 & $43 \%$ & $<0.0001$ & 55,3 \\
\hline \multicolumn{7}{|c|}{ Pterostilbene } \\
\hline I & 100 & 9.55 & 12.16 & $79 \%$ & 0.005 & 69,9 \\
\hline 4 & 20 & 10.43 & 11.26 & $93 \%$ & 0.336 & 54,0 \\
\hline 4 & 40 & 10 & 11.26 & $89 \%$ & 0.035 & 65,1 \\
\hline 4 & 100 & 8.07 & 11.26 & $72 \%$ & $<0.0001$ & 51,1 \\
\hline 6 & 20 & 13.72 & 15.32 & $90 \%$ & 0.058 & 55,1 \\
\hline 6 & 40 & $|4.4|$ & 15.32 & $94 \%$ & 0.142 & $5 I, 4$ \\
\hline 6 & 100 & 10.98 & 15.32 & $72 \%$ & $<0.001$ & 51,0 \\
\hline \multicolumn{7}{|c|}{ Desoxyrhapontigenin } \\
\hline 1 & 100 & 10.64 & 12.16 & $88 \%$ & 0.12 & 61,1 \\
\hline 3 & 100 & 11.31 & 15.37 & $74 \%$ & $<0.0001$ & 36,0 \\
\hline 3 & 200 & 11.61 & 15.37 & $76 \%$ & $<0.0001$ & 44,1 \\
\hline 4 & 20 & 10.2 & 11.26 & $91 \%$ & 0.12 & 74,0 \\
\hline 4 & 40 & 10.38 & 11.26 & $92 \%$ & 0.11 & 71,2 \\
\hline 4 & 100 & 9.44 & 11.26 & $84 \%$ & 0.003 & 50,0 \\
\hline 6 & 20 & 14.18 & 15.32 & $93 \%$ & 0.494 & 54,3 \\
\hline 6 & 40 & $|5.3|$ & 15.32 & $100 \%$ & 0.48 & 59,1 \\
\hline 6 & 100 & 13.63 & 15.32 & $89 \%$ & 0.027 & 46,9 \\
\hline \multicolumn{7}{|c|}{ Pinostilbene } \\
\hline 1 & 100 & 13.6 & 12.16 & $112 \%$ & 0.062 & 68,1 \\
\hline 2 & 20 & 10.04 & 11.05 & $91 \%$ & 0.266 & 55,0 \\
\hline 2 & 40 & 11.53 & 11.05 & $104 \%$ & 0.319 & 42,5 \\
\hline 3 & 100 & 16.9 & 15.37 & $110 \%$ & 0.448 & 41,0 \\
\hline 3 & 200 & 15.23 & 15.37 & $99 \%$ & 0.411 & 42,5 \\
\hline 6 & 20 & 14.24 & 15.32 & $93 \%$ & 0.319 & 56,3 \\
\hline 6 & 40 & 17.33 & 15.32 & $113 \%$ & 0.085 & 60,0 \\
\hline 6 & 100 & 16.24 & 15.32 & $106 \%$ & 0.724 & 51,2 \\
\hline
\end{tabular}


Table I: Adult survival statistics for all trials. (Continued)

\begin{tabular}{|c|c|c|c|c|c|c|}
\hline Resve & & & & & & \\
\hline I & 100 & 12.18 & 12.16 & $100 \%$ & 0.867 & 70,3 \\
\hline 2 & 20 & 11.85 & 11.05 & $107 \%$ & 0.182 & 48,4 \\
\hline 2 & 40 & 10.53 & 11.05 & $95 \%$ & 0.924 & 55,0 \\
\hline 3 & 100 & 16.64 & 15.37 & $108 \%$ & 0.435 & 30,4 \\
\hline 3 & 200 & 17 & 15.37 & $111 \%$ & 0.337 & 38,0 \\
\hline 6 & 20 & 14.65 & 15.32 & $96 \%$ & 0.804 & 54,5 \\
\hline 6 & 40 & 16.17 & 15.32 & $106 \%$ & 0.501 & 59,0 \\
\hline 6 & 100 & 17.55 & 15.32 & $115 \%$ & 0.055 & 58,0 \\
\hline Picea & & & & & & \\
\hline 2 & 20 & 11.72 & 11.05 & $106 \%$ & 0.362 & 44,0 \\
\hline 2 & 40 & 11.58 & 11.05 & $105 \%$ & 0.5 & 37,9 \\
\hline 3 & 100 & 17.78 & 15.37 & $116 \%$ & 0.094 & 55,1 \\
\hline 3 & 200 & 17.5 & 15.37 & $114 \%$ & 0.166 & 55,1 \\
\hline 6 & 20 & |3.7| & 15.32 & $89 \%$ & 0.231 & 50,1 \\
\hline 6 & 40 & 15.63 & 15.32 & $102 \%$ & 0.75 & 50,1 \\
\hline 6 & 100 & 16.6 & 15.32 & $108 \%$ & 0.146 & 50,1 \\
\hline
\end{tabular}

ited a modest inhibitory effect on $g l p-1$ (q485) germline tumor growth in two of three experiments $(-4 \%,-7 \%$, $19 \% ; p \leq 0.05$ for trials 2, 3, t-test vs untreated). At $100 \mu \mathrm{M}$ dose, the methoxylated stilbenes resveratrol trimethylether and desoxyrhapontigenin were also associated with consistent reductions in tumor size. Pterostilbene also modestly reduced tumor size, and this effect was statistically significant in 2 of 3 trials $(p \leq 0.05$, t-test vs untreated). In contrast, resveratrol had a negligible effect on gld-1(q485) germline tumor size which was not statistically significant $(-4 \%, p=0.35)$. As expected from its known antiproliferative effect, FUDR treatment also appeared to decrease tumor size, although the effect was statistically significant in only 2 of 3 trials.

To better quantify the effects of stilbenes and FUDR on gld-1(q485) tumor growth, two stilbenes, resveratrol and pterostilbene, and FUDR were examined at a series of additional doses between 50-400 $\mu \mathrm{M}$. Over this range of concentrations, both FUDR and pterostilbene were associated with $10-20 \%$ reduction in tumor area (Figure 5A, B). The effects of pterostilbene were somewhat variable between trials, and were statistically significant for 3 of 4 experiments at both the 200 and $400 \mu \mathrm{M}$ dose. The 200 and $400 \mu \mathrm{M}$ doses of FUDR were consistently associated with smaller tumor area and these effects were significant in all 4 trials. Resveratrol at the 200 and $400 \mu \mathrm{M}$ dose was not associated with statistically significant reductions in tumor area for any of the three trials conducted. These data demonstrate that the enhanced bioactivity of methoxylated stilbenes in vivo in C. elegans can be detected as a reduction in $g l p-1(q 485)$ tumor area as well as by adult toxicity.

The differential bioactivities of methoxylated and hydroxylated stilbenes in C. elegans adults might reflect differ- ences in uptake or bioavailability. In general, intact $C$. elegans adults take up compounds relatively poorly due to their thick, impermeable cuticle. This impermeability has impeded pharmacodynamic analysis in this organism. However, steady-state levels of compounds can be measured in treated animals to provide one measure of pharmacodynamics. To assess whether differential uptake correlated with bioactivity in C. elegans, steady-state levels for pterostilbene, resveratrol-trimethylether and desoxyrhapontigenin were determined in animals treated for 2 days with $100 \mu \mathrm{M}$ of each compound in the culture medium. The levels of these stilbenes in C. elegans nematodes ranged from $20-80 \mu \mathrm{M}$, indicating relatively efficient uptake (Figure 6). These concentrations were within the range of serum stilbene levels detected after dietary stilbene supplementation with human volunteers (approx. $2 \mu \mathrm{M})$ and rabbits $(42.8 \mu \mathrm{M})$ [29]. It is also within the dose range where resveratrol was observed to induce dilation of isolated retinal arterioles $(50 \mu \mathrm{M})$ [30]. These three compounds, while all methoxylated, exhibited different levels of toxicity in adult C. elegans. In treated worms, levels of pterostilbene were slightly elevated compared with resveratrol-trimethylether and desoxyrhapontigenin (Figure 6). However, the most toxic compound in this group, resveratrol-trimethylether, was present at less than one-third the level of pterostilbene. These data suggest that relative bioactivity did not solely reflect steady-state levels in intact animals, although, this experiment does not directly compare the pharmacokinetic profile of each compound in C. elegans.

This study compared the effects of methoxylated and hydroxylated stilbenes on adult lifespan and tumor growth in C. elegans nematodes. In this organism, stilbene methoxylation was associated with toxicity under chronic treatment conditions and reduced tumor size in vivo. 

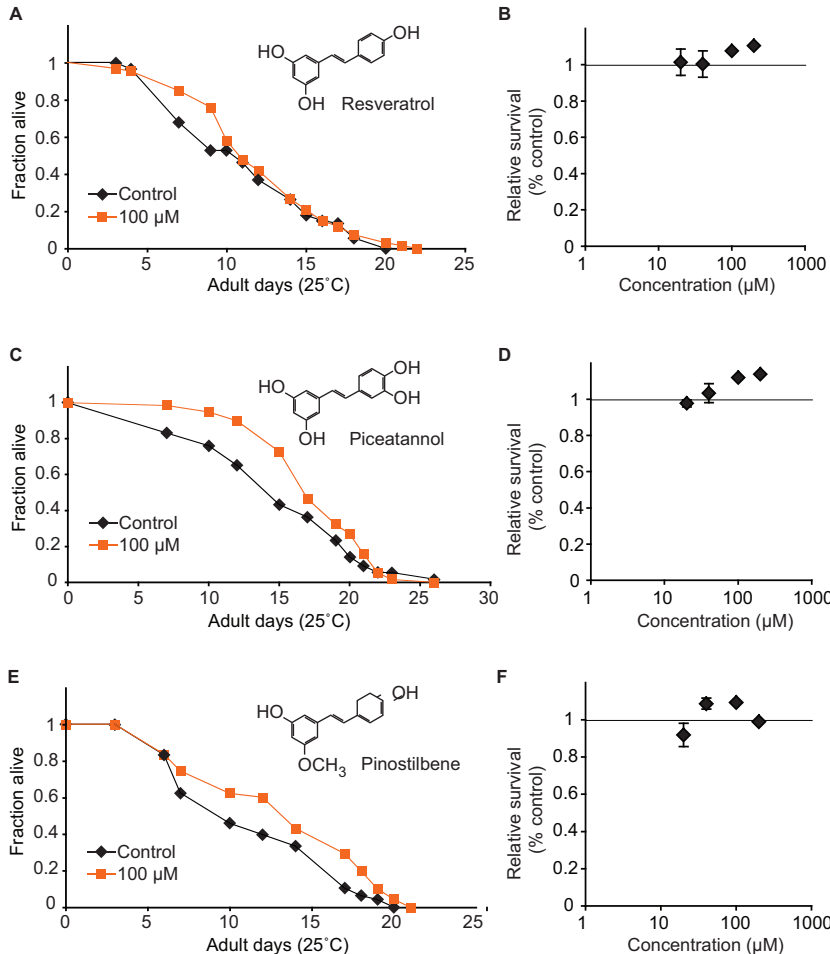

Figure 2

Hydroxylated and one monomethoxylated stilbene had modest beneficial effects on adult $C$. elegans survival. $(A, C, E)$ Representative survival curves of adult sterile fem-l (hcl 7) animals treated with indicated doses of each compound or vehicle in the medium. Experiments for each compound were conducted concurrently for all doses shown. (B, D, F) Summary data from $n=1-3$ independent experiments per concentration tested, showing doseresponse for each compound. Complete survival statistics are presented in Table I.

Overall, this study corroborated other findings of enhanced bioactivities in methoxylated versus hydroxylated stilbenes in other experimental systems [31]. However, ours is the first study to systematically evaluate the effects of stilbene methoxylation on bioactivity in a whole organism, using C. elegans.

The mechanisms for enhanced bioactivity of methoxylated stilbenes may reflect increased biostability in vivo due to reduced efficiency of metabolic modification leading to excretion. In the case of another group of compounds, the flavonoids, substituting the hydroxyl groups with methoxy groups was shown to improve flavonoid stability in cultured cells and liver microsomes by blocking metabolic modification and excretion [12,11]. In mammals, hydroxylated flavonoids were shown to undergo glucuronidation by hepatic enzymes to promote excretion [11]. Thus, methoxylation may block this mod-
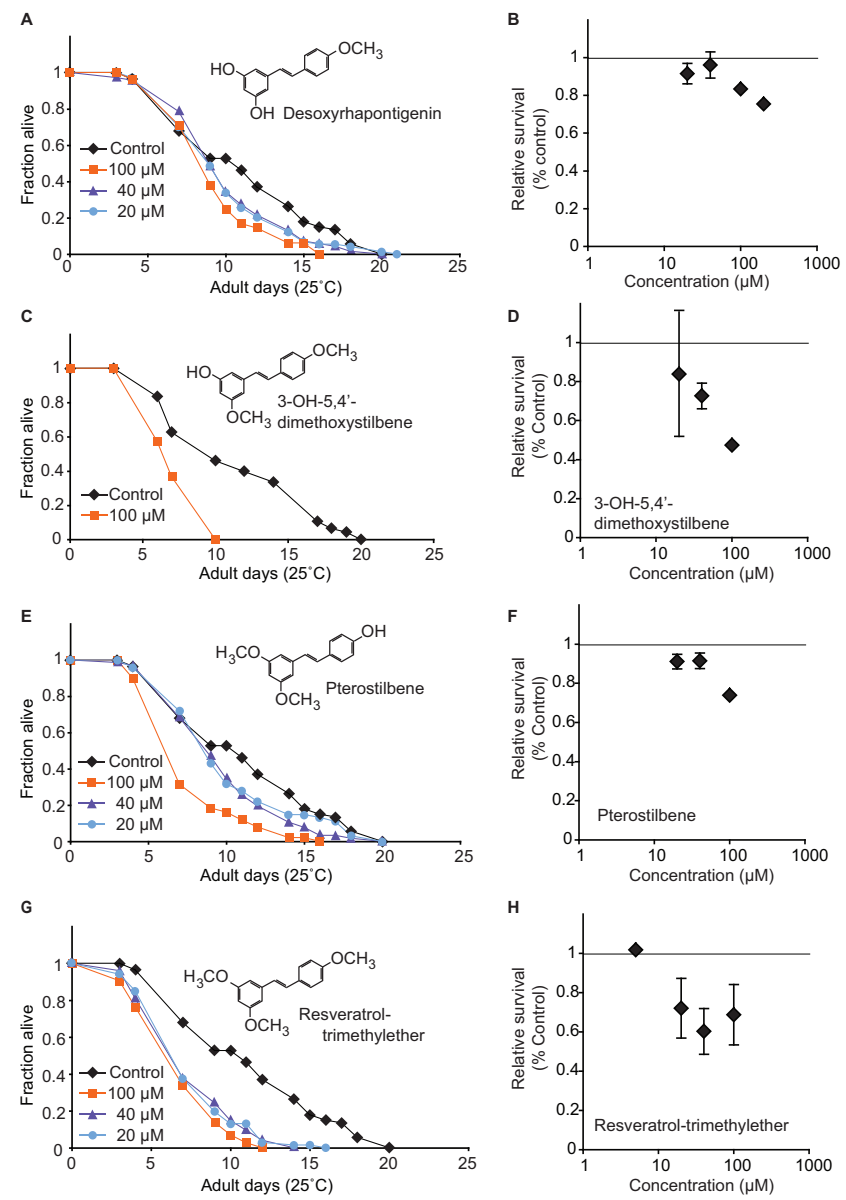

Figure 3

Methoxylated stilbenes were toxic to $C$. elegans adults. (A, C, E, G) Representative survival curves of femI (hcl 7) sterile adults treated with compounds as indicated from the first day of adulthood. (B, D, F, H) Summary data for independent experiments at indicated concentrations. Complete survival statistics presented in Table I.

ification and may also promote biostability. Indeed, one naturally methoxylated stilbene, pterostilbene, is cytotoxic in several cellular systems [32]. In contrast, the hydroxylated stilbene, resveratrol, has also been shown to be cytotoxic, but is poorly retained in plasma compared with pterostilbene [33]. Thus, methoxylation differences between pterostilbene and resveratrol may improve bioactivity by increasing in vivo stability.

The basis for the toxicity and antiproliferative activities of methyoxylated stilbenes in C. elegans adults is not known. The somatic cells of $C$. elegans adults are entirely postmitotic, ruling out antiproliferative effects as the basis for methoxylated stilbene toxicity. However, the toxicity and antitumor activities of methoxylated stilbenes in C. elegans adults may reflect interactions with common biolog- 
A

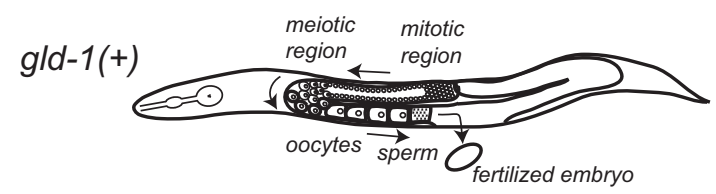

$g l d-1(q 485)$

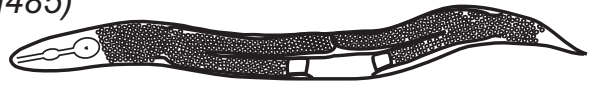

B

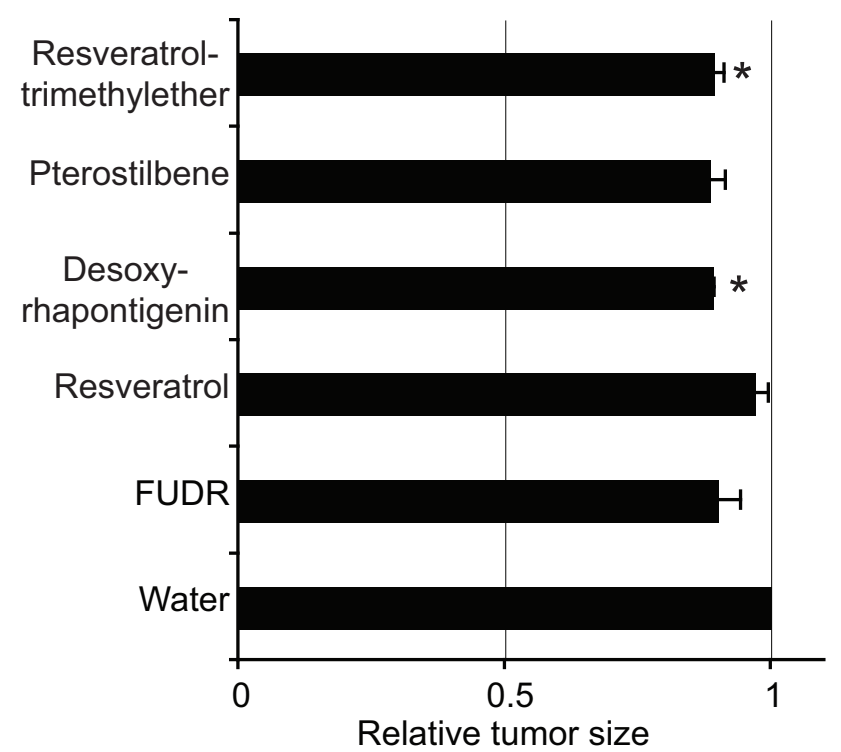

Figure 4

Methoxylated stilbenes, desoxyrhapontigenin and resveratrol-trimethyether, demonstrated modest anti-tumor activity in vivo. (A) Upper right, cartoon of normal germ cell proliferation and differentiation in gld-I $(+)$ animals. Below, cartoon of aberrant germline tumors formed in $g / d-I(q 485)$ animals. In tumor-containing $g / p-I(q 485)$ adults, DAPI staining allows visualization and quantification of germline tumors as the fluorescent area filling the body. (B) Mean germline tumor size (area \pm SEM) relative to control in $g l p$ $I(q 485)$ adults treated with $100 \mu M$ doses of indicated stilbenes or FUDR. Results are average from at least 3 independent experiments. In each experiment, tumor area was determined for 12-47 individuals per compound (average \pm $S D=23.1 \pm 10.12$ animals). Significance was judged in paired t-test (2-tailed); $* p<0.05$ versus untreated.

ical targets. Studies in other experimental systems show that stilbenes are potent antioxidants [34,35]. However, resveratrol and pterostilbene, which have strikingly different activity in vivo in adult $C$. elegans, demonstrated similar antioxidant activity in vitro, suggesting that antioxidant activity is not the underlying reason for the difference in stilbene bioactivities in C. elegans. Stilbenes are also known to inhibit specific cellular enzymes, such as cyto- chrome P450 and COX enzymes. One study has compared the relative inhibition of mammalian CYP1 cytochrome P450 enzymes by pterostilbene, pinostilbene and desoxyrhapontigenin [5]. In this study, these three methoxylated stilbenes demonstrated increased CYP1 inhibition relative to resveratrol, in partial agreement with our results. However, pinostilbene demonstrated slightly stronger CYP1 inhibition than pterostilbene and desoxyrhapontigenin, a finding not consistent with our C. elegans toxicity data. Stilbenes also inhibit COX-1 and -2 monooxygenases. C. elegans nematodes do not have any obvious COX-1 or -2 homologs, although the C. elegans genome sequence does contain numerous monooxygenases that might have similar mechanisms of action to mammalian COX-1 and - 2 enzymes. Further characterization of the in vivo targets for methyoxylated stilbenes in $C$. elegans may provide insight into other bioactivities of these compounds in human cells.

\section{Conclusion}

Stilbene methoxylation was associated with increased in vivo bioactivity as tested by toxicity and tumor growth in C. elegans. In general, increasing degree of methoxylation was correlated with increasing bioactivity, with the exception of pinostilbene. However, methoxylation and toxicity were not correlated with steady-state levels of these compounds in treated animals. These findings suggest that methoxylation enhances bioactivity possibly through increased interactions with in vivo targets. A working hypothesis to account for these results is that stilbene methoxylation protects the compound from conjugation and subsequent excretion, thereby increasing biostability and bioavailability in C. elegans.

\section{Methods}

\section{C. elegans strains and culture conditions}

C. elegans strains were maintained at $15^{\circ} \mathrm{C}$ on NGM agar medium with a live, slow-growing OP50 bacterial lawn for a food source following standard protocols [36]. The following mutant strains were used in this study: BA17, fem-1(hc17), and JK1466, gld-1(q485)/dpy-5(e61) unc13(e51). Strains were obtained from the Canorhabditis Genetics Center at the University of Minnesota.

\section{Chemical sources and methoxylated stilbene preparation}

Resveratrol and picetannol were obtained from commercial sources (Sigma-Aldrich, Inc. and Calbiochem-Novobiochem Corp., respectively). The methoxylated stilbenes pinostilbene, desoxyrhapontigenin, 3-hydroxy-5,4'dimethoxystilbene and pterostilbene (Figure 1) were synthesized by partial methylation of trans-resveratrol as previously reported [34]. The methoxylated stilbenes were purified by preparative layer chromatography (Merck Silica gel 60 F254; EMD Chemicals Inc., Gibbstown, NJ) using the developing solvent chloroform:methanol (96:4) 
A
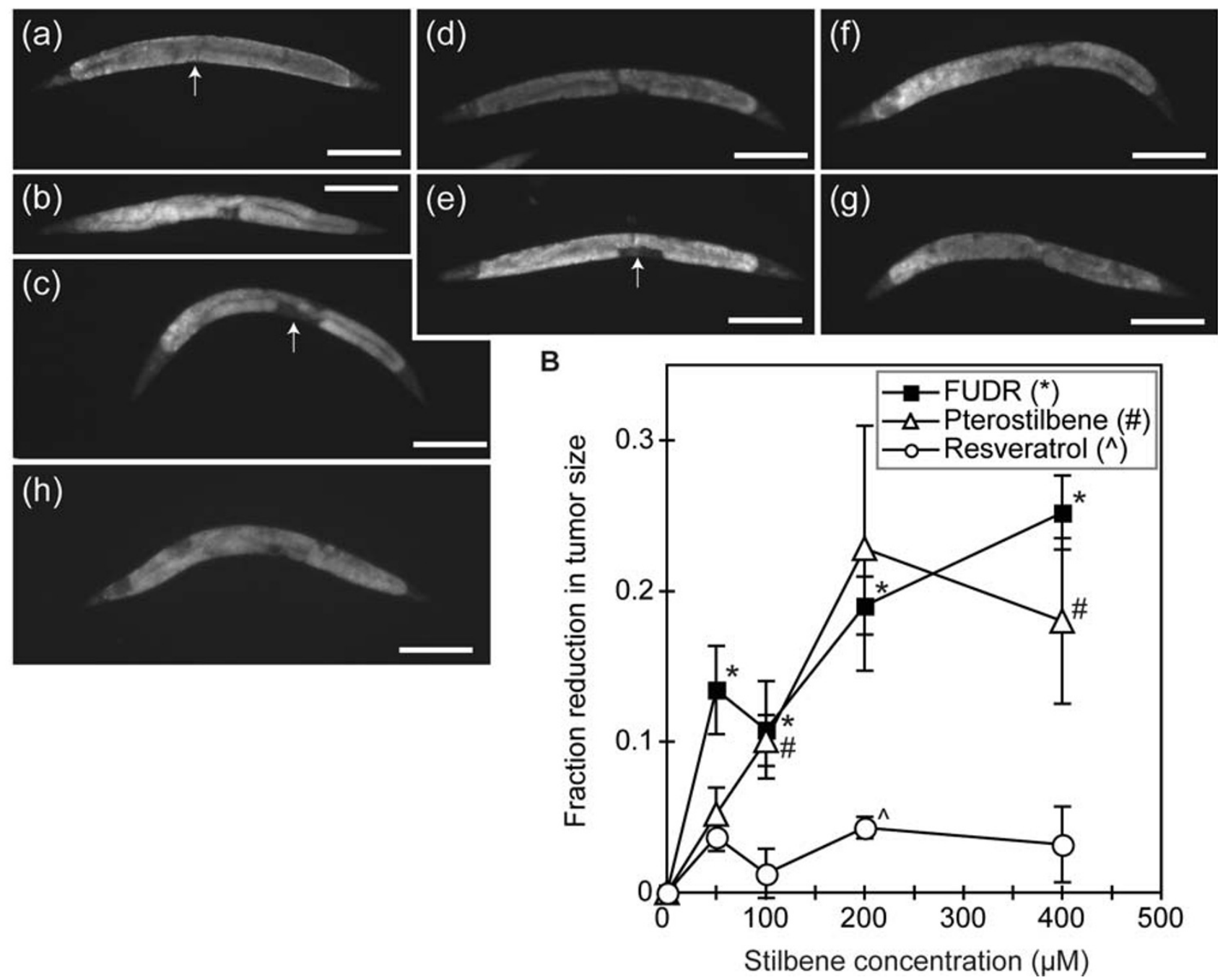

Figure 5

Titration of germline tumor growth suppression by FUDR, resveratrol and pterostilbene. (A) Representative images of DAPI-stained glp-I (q485) animals treated for four days with $100 \mu \mathrm{M}$ or $400 \mu \mathrm{M}$ doses of FUDR, resveratrol and pterostilbene. The inhibitory effect of FUDR and pterostilbene on tumor size is particularly evident near the central region of the animal where the reflexed arms of the distal gonad meet (arrow in (a), (c) and (e)). (a, h) vehicle-treated controls. (h) is from the same experimental series as (c); (a) is from the same experimental series as other images; (b, c), FUDR at I00 $\mu M(b)$ and $400 \mu \mathrm{M}$ (c); (d, e), pterostilbene at $100 \mu \mathrm{M}$ (d) and $400 \mu \mathrm{M}$ (e); (f, g) resveratrol at I00 $\mu \mathrm{M}$ (f) and $400 \mu \mathrm{M}$ (g). Scale bar = 250 $\mu$ M. (B) Summary of results from three independent experiments for each compound at indicated doses. Each concentration was tested in at least 3 independent trials and a total of 36-100 animals were measured per compound dose in all experiments. Significance was judged by a paired t-test (2-tailed); $*, \#,{ }^{\wedge} p<0.05$ versus untreated for FUDR-, pterostilbene- or resveratroltreated animals, respectively.

with $1 \%$ formic acid. The Rf values for pterostilbene, 3 hydroxy-5,4'-dimethoxystilbene, pinostilbene and desoxyrhapontigenin are $0.61,0.57,0.28$, and 0.20 , respectively. These stilbenes were identified from their ${ }^{1} \mathrm{H}-\mathrm{NMR}$ spectra (Bruker $400 \mathrm{MHz}$; Bruker, Billerica, MA). All stilbenes were dissolved in ethanol to $25 \mathrm{mg} / \mathrm{mL}$ before use. 5-Fluoro-deoxyuracil (Sigma Chemical Co.) was dissolved to $1 \mathrm{mg} / \mathrm{mL}$ in sterile water and stored frozen at $20^{\circ} \mathrm{C}$ until use. For treating C. elegans adults, each com- pound was added in a $200 \mu \mathrm{L}$ volume to the top of a 1-day old bacterial lawn grown on NGM medium to final concentrations as indicated. Compounds were allowed 2 hours to diffuse through the agar medium before animals were added.

\section{Survival assays}

For survival analyses, synchronized populations of sterile fem-1 (hc17) hermaphrodites were obtained by first trans- 


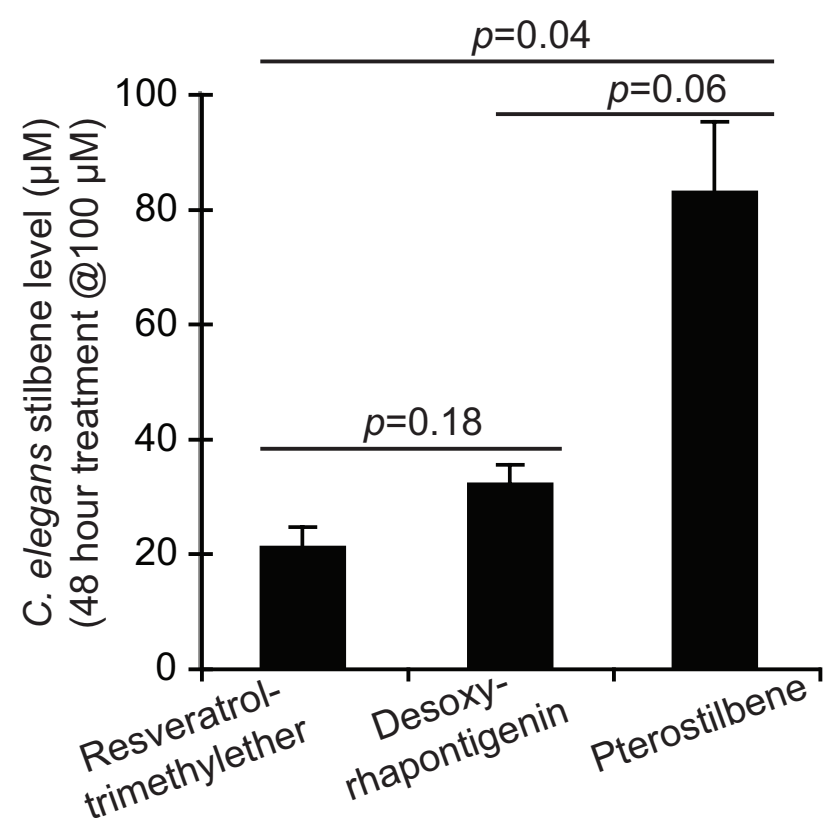

Figure 6

Steady-state levels of three stilbene compounds in treated $C$. elegans adults did not correlate with toxicity. Animals were treated for two days with a toxic dose $(100 \mu \mathrm{M})$ of indicated compound in the medium. After collection, stilbene levels determined as described in methods. Compounds were measured in two independent samples for each treatment with paired negative control. Averages and SDs between both measurements are shown; statistical significance was determined by t-test.

ferring several fertile hermaphrodites to a fresh plate at $25^{\circ} \mathrm{C}$ and allowing them to lay eggs for several hours. The parent hermaphrodites were removed and embryos were allowed to develop into sterile adults at $25^{\circ} \mathrm{C}$, which takes approximately 48 hours [17]. On the first day of adulthood, referred to as day 0 , sterile adults were transferred onto fresh media supplemented with stilbenes at indicated concentrations, and maintained at $25^{\circ} \mathrm{C}$. Adult survival was scored every 2-3 days by the animal's ability to move in response to a gentle mechanical stimulus. Statistical analysis of survival was performed using the JMP software package.

\section{Germline tumor growth assays}

The gld-1(q485) mutation causes a defect in oocyte development that results in growth of germline tumors that fill the somatic gonad, eventually leading to the animal's death $[20,21]$. To examine the effects of the stilbenes on gld-1(q485) germline tumors, day 0 adults were placed on stilbene-containing medium in the presence of OP50 bacteria as food source. After 4 days at $20^{\circ} \mathrm{C}$, animals were fixed in methanol and germcell nuclei were visualized by staining with DAPI. Images were collected with the entire animal in view using the $4 \times$ objective on a Nikon E800 microscope equipped with a Hamamatsu Orca ER CCD camera using OpenLab imaging software. NIH Image J software was used to measure germline tumor growth in treated and untreated animals. Tumor area was measured by outlining the entire DAPI-stained tumor.

\section{Determination of stilbene uptake}

Uptake was determined for three stilbenes (desoxyrhapontigenin, pterostilbene and resveratrol-trimethylether) by measuring the steady-state levels of each stilbene in adult fem-1(hc17) hermaphrodites treated under the same conditions as those used for survival assays. After a 2-day treatment on stilbene-supplemented medium, animals were collected into eppendorf tubes, washed with sterile $\mathrm{M} 9$ and allowed to settle under a minimal volume of liquid. The samples were frozen at $-80^{\circ} \mathrm{C}$ for storage. For the analysis of stilbenes, samples were thawed in ice and homogenized in $300 \mu \mathrm{L}$ of $0.2 \mathrm{M}$ phosphate buffer then centrifuged $\left(4^{\circ} \mathrm{C}, 4000 \mathrm{rpm}, 15 \mathrm{~min}\right)$. The supernatant was collected and the pellet was homogenized further with fresh $300 \mu \mathrm{L}$ of $0.2 \mathrm{M}$ phosphate buffer then centrifuged. The supernatants were combined and loaded on an Oasis ${ }^{\circledast}$ HLB solid phase extraction cartridge (Waters Corporation, Milford, MA) that has been preconditioned with $0.2 \mathrm{M}$ phosphate buffer. The stilbenes were eluted with 2 $\mathrm{mL}$ of methanol and collected in a vial. The eluate was dried under a stream of nitrogen, redissolved in $1 \mathrm{~mL}$ of ethyl acetate, filtered $(0.2 \mu \mathrm{m}$ nylon filter $)$ then dried again under a stream of nitrogen. The dried sample was treated with $30 \mu \mathrm{L}$ of $\mathrm{N}, \mathrm{O}-$ bis [trimethylsilyl]trifluoroacetamide:dimethylformamide (BSTFA: DMF, 1:1; Pierce Biotechnology, Inc., Rockford, IL) and heated at $70^{\circ} \mathrm{C}$ for $45 \mathrm{~min}$. Derivatized sample was analyzed for stilbenes by gas chromatography-mass spectrometry (GC-MS). GC-MS was performed on a JEOL GCMate II spectrometer (JEOL USA Inc., Peabody, MA) in tandem with an Agilent $6890 \mathrm{~N}$ gas chromatograph (Agilent Technologies, Santa Clara, CA) using a J\&W DB-5 capillary column $(0.25 \mathrm{~mm}$ internal diameter, $0.25 \mu \mathrm{m}$ film thickness, $30 \mathrm{~m}$ length; Agilent Technologies). The GC temperature program was: initial $190^{\circ} \mathrm{C}$, increased to $240^{\circ} \mathrm{C}$ at $25^{\circ} \mathrm{C} / \mathrm{min}$ rate and held at this temp for $14 \mathrm{~min}$, then finally increased to $300^{\circ} \mathrm{C}$ at the rate of $25^{\circ} \mathrm{C} / \mathrm{min}$ and held at this temperature for 1 $\mathrm{min}$. The carrier gas was ultrahigh purity helium, at $1 \mathrm{~mL} /$ min flow rate. The injection port, GC-MS interface and ionization chamber were at 250,230 and $230^{\circ} \mathrm{C}$, respectively. The volume of injection was $1 \mu \mathrm{L}$, splitless injection. Mass spectrum was acquired in positive, electron impact $(70 \mathrm{eV})$, selected ion monitoring mode. Resveratrol-trimethylether (retention time $10.6 \mathrm{~min}$ ) was monitored at $\mathrm{m} / \mathrm{z} 270,239$ and 196; desoxyrhapontigenin (retention time $13.1 \mathrm{~min}$ ) was monitored at $\mathrm{m} / \mathrm{z} 328,313$ and 206; pterostilbene (retention time $13.6 \mathrm{~min}$ ) was 
monitored at $m / z$ 386, 371 and 297. Quantitation was performed from calibration curve of authentic stilbene samples. GC-MS analyses were in duplicates. For conversion from $\mathrm{pg}$ /worm to molarity concentration in vivo, the volume of a wildtype hermaphrodite on day 4 of adulthood was taken to be $4.5 \mu \mathrm{L}$, as previously determined [37].

\section{Authors' contributions}

MAW and CAW conceived of and designed the C. elegans experiments, which were performed entirely by MAW. AMR prepared the stilbene derivatives and performed the uptake determinations. CAW, MAW and AMR prepared the manuscript. All authors have read and approved the manuscript.

\section{Acknowledgements}

Strains were obtained from the Caenorhabditis Genetics Center, housed by the University of Minnesota and funded by the National Center for Research Resources of the National Institutes of Health. We thank J. Joseph, N. Greig and D. Longo for helpful discussions. This work was funded in part by the Intramural Research Program of the National Institute on Aging, NIH (ZOI AG000320-06 LN [2007] Genetic and environmental factors that regulate aging and longevity).

\section{References}

I. Bosetti C, Rossi M, McLaughlin JK, Negri E, Talamini R, Lagiou P, Montella M, Ramazzotti V, Franceschi S, LaVecchia C: Flavonoids and the risk of renal cell carcinoma. Cancer Epidemiol Biomarkers Prev 2007, I6(I):98-I0I.

2. Rimando AM, Nagmani R, Feller DR, Yokoyama W: Pterostilbene, a new agonist for the peroxisome proliferator-activated receptor alpha-isoform, lowers plasma lipoproteins and cholesterol in hypercholesterolemic hamsters. J Agric Food Chem 2005, 53(9):3403-3407.

3. Rossi M, Garavello W, Talamini R, Negri E, Bosetti C, Dal Maso L, Lagiou P, Tavani A, Polesel J, Barzan L, Ramazzotti V, Franceschi S, La $V$ ecchia $C$ : Flavonoids and the risk of oral and pharyngeal cancer: a case-control study from Italy. Cancer Epidemiol Biomarkers Prev 2007, I6(8): |62|-|625.

4. Suresh Babu K, Tiwari AK, Srinivas PV, Ali AZ, China Raju B, Rao JM: Yeast and mammalian alpha-glucosidase inhibitory constituents from Himalayan rhubarb Rheum emodi Wall.ex Meisson. Bioorganic \& medicinal chemistry letters 2004, I 4( I 4):384 I-3845.

5. Mikstacka R, Przybylska D, Rimando AM, Baer-Dubowska W: Inhibition of human recombinant cytochromes P450 CYPIAI and CYPIB I by trans-resveratrol methyl ethers. Molecular nutrition \& food research 2007, 5 I(5):5I7-524.

6. Remsberg CM, Yanez JA, Ohgami Y, Vega-Villa KR, Rimando AM, Davies NM: Pharmacometrics of pterostilbene: preclinical pharmacokinetics and metabolism, anticancer, antiinflammatory, antioxidant and analgesic activity. Phytother Res 2007, 22:169-179.

7. Pari L, Satheesh MA: Effect of pterostilbene on hepatic key enzymes of glucose metabolism in streptozotocin- and nicotinamide-induced diabetic rats. Life sciences 2006 , 79(7):64|-645.

8. Bauer JH, Goupil S, Garber GB, Helfand SL: An accelerated assay for the identification of lifespan-extending interventions in Drosophila melanogaster. Proc Natl Acad Sci U S A 2004, I 0 I(35): I 2980- I 2985.

9. Wood JG, Rogina B, Lavu S, Howitz K, Helfand SL, Tatar M, Sinclair $D$ : Sirtuin activators mimic caloric restriction and delay ageing in metazoans. Nature 2004, 430(7000):686-689.

10. Lagouge M, Argmann C, Gerhart-Hines Z, Meziane H, Lerin C, Daussin F, Messadeq N, Milne J, Lambert P, Elliott P, Geny B, Laakso $M$, Puigserver $P$, Auwerx J: Resveratrol improves mitochondrial function and protects against metabolic disease by activating SIRTI and PGC-I alpha. Cell 2006, I27(6): I 109-I I 22.

II. Wen X, Walle T: Methylation protects dietary flavonoids from rapid hepatic metabolism. Xenobiotica; the fate of foreign compounds in biological systems 2006, 36(5):387-397.

12. Wen X, Walle T: Methylated flavonoids have greatly improved intestinal absorption and metabolic stability. Drug metabolism and disposition: the biological fate of chemicals 2006, 34(10): 1786-1792.

13. Walle T, Ta N, Kawamori T, Wen X, Tsuji PA, Walle UK: Cancer chemopreventive properties of orally bioavailable flavonoids--methylated versus unmethylated flavones. Biochemical pharmacology 2007, 73(9): I 288- 1296.

14. Menzel R, Yeo HL, Rienau S, Li S, Steinberg CE, Sturzenbaum SR: Cytochrome P450s and short-chain dehydrogenases mediate the toxicogenomic response of PCB52 in the nematode Caenorhabditis elegans. Journal of molecular biology 2007, 370(I): $1-13$.

15. Wilson MA, Shukitt-Hale B, Kalt W, Ingram DK, Joseph JA, Wolkow $C A$ : Blueberry polyphenols increase lifespan and thermotolerance in Caenorhabditis elegans. Aging Cell 2006, 5(I):59-68.

16. Wu Y, Wu Z, Butko P, Christen Y, Lambert MP, Klein WL, Link CD, Luo Y: Amyloid-beta-induced pathological behaviors are suppressed by Ginkgo biloba extract EGb $76 \mathrm{I}$ and ginkgolides in transgenic Caenorhabditis elegans. J Neurosci 2006, 26(50): $|3| 02-|3| \mid 3$.

17. Doniach T, Hodgkin J: A sex-determining gene, fem-I, required for both male and hermaphrodite development in Caenorhabditis elegans. Developmental biology 1984, I 06(I):223-235.

18. Nelson GA, Lew KK, Ward S: Intersex, a temperature-sensitive mutant of the nematode Caenorhabditis elegans. Developmental biology 1978, 66(2):386-409.

19. Mikstacka R, Rimando AM, Szalaty K, Stasik K, Baer-Dubowska W: Effect of natural analogues of trans-resveratrol on cytochromes P450 IA2 and 2EI catalytic activities. Xenobiotica; the fate of foreign compounds in biological systems 2006, 36(4):269-285.

20. Francis R, Barton MK, Kimble J, Schedl T: gld-I, a tumor suppressor gene required for oocyte development in Caenorhabditis elegans. Genetics 1995, 139(2):579-606.

21. Francis R, Maine E, Schedl T: Analysis of the multiple roles of gld$I$ in germline development: interactions with the sex determination cascade and the glp-I signaling pathway. Genetics 1995, I39(2):607-630.

22. Cardile V, Chillemi R, Lombardo L, Sciuto S, Spatafora C, Tringali C: Antiproliferative activity of methylated analogues of $\mathrm{E}$ - and Z-resveratrol. Zeitschrift fur Naturforschung 2007, 62(3-4): I 89-195.

23. Chillemi R, Sciuto S, Spatafora C, Tringali C: Anti-tumor properties of stilbene-based resveratrol analogues: recent results. Nat Prod Comm 2007, 2(4):499-5। 3.

24. Mgbonyebi OP, Russo J, Russo IH: Antiproliferative effect of synthetic resveratrol on human breast epithelial cells. International journal of oncology 1998, I2(4):865-869.

25. Nigro P, Bloise E, Turco MC, Skhirtladze A, Montoro P, Pizza C, Piacente S, Belisario MA: Antiproliferative and pro-apoptotic activity of novel phenolic derivatives of resveratrol. Life sciences 2007, 8I (II):873-883

26. Heidelberger C, Chaudhuri NK, Danneberg P, Mooren D, Griesbach L, Duschinsky R, Schnitzer RJ, Pleven E, Scheiner J: Fluorinated pyrimidines, a new class of tumour-inhibitory compounds. Nature 1957, 179(456I):663-666.

27. Santi DV, McHenry CS: 5-Fluoro-2'-deoxyuridylate: covalent complex with thymidylate synthetase. Proc Natl Acad Sci U S A 1972, 69(7): 1855-1857.

28. Hosono R: Sterilization and growth inhibition of Caenorhabditis elegans by 5-fluorodeoxyuridine. Experimental gerontology 1978, 13(5):369-374

29. Wenzel E, Somoza V: Metabolism and bioavailability of transresveratrol. Molecular nutrition \& food research 2005, 49(5):472-48।.

30. Nagaoka T, Hein TW, Yoshida A, Kuo L: Resveratrol, a component of red wine, elicits dilation of isolated porcine retinal arterioles: role of nitric oxide and potassium channels. Investigative ophthalmology \& visual science 2007, 48(9):4232-4239.

31. Likhitwitayawuid K, Sornsute A, Sritularak B, Ploypradith P: Chemical transformations of oxyresveratrol (trans-2,4,3',5'-tetrahydroxystilbene) into a potent tyrosinase inhibitor and a 
strong cytotoxic agent. Bioorganic \& medicinal chemistry letters 2006, I 6(2I):5650-5653.

32. Rimando AM, Pezzuto JM, Farnsworth NR, Santisuk T, Reutrakul V, Kawanishi K: New lignans from Anogeissus acuminata with HIV-I reverse transcriptase inhibitory activity. Journal of natural products 1994, 57(7):896-904.

33. Ferrer P, Asensi M, Segarra R, Ortega A, Benlloch M, Obrador E, Varea MT, Asensio G, Jorda L, Estrela JM: Association between pterostilbene and quercetin inhibits metastatic activity of B 16 melanoma. Neoplasia (New York, NY) 2005, 7(I):37-47.

34. Rimando AM, Cuendet M, Desmarchelier C, Mehta RG, Pezzuto JM, Duke SO: Cancer chemopreventive and antioxidant activities of pterostilbene, a naturally occurring analogue of resveratrol. J Agric Food Chem 2002, 50( I 2):3453-3457.

35. Stivala LA, Savio M, Carafoli F, Perucca P, Bianchi L, Maga G, Forti L, Pagnoni UM, Albini A, Prosperi E, Vannini V: Specific structural determinants are responsible for the antioxidant activity and the cell cycle effects of resveratrol. J Biol Chem 200I, 276(25):22586-22594.

36. Brenner S: The genetics of Caenorhabditis elegans. Genetics 1974, 77:71-94.

37. Hirose T, Nakano Y, Nagamatsu Y, Misumi T, Ohta H, Ohshima Y: Cyclic GMP-dependent protein kinase EGL-4 controls body size and lifespan in C elegans. Development (Cambridge, England) 2003, I30(6): 1089-1099.

Publish with Bio Med Central and every scientist can read your work free of charge

"BioMed Central will be the most significant development for disseminating the results of biomedical research in our lifetime. "

Sir Paul Nurse, Cancer Research UK

Your research papers will be:

- available free of charge to the entire biomedical community

- peer reviewed and published immediately upon acceptance

- cited in PubMed and archived on PubMed Central

- yours - you keep the copyright

Submit your manuscript here:

http://www.biomedcentral.com/info/publishing_adv.asp
BioMedcentral 